Mots. Les langages du politique

$81 \mid 2006$

Suisse, laboratoire politique européen ?

\title{
Marie-Claire Caloz-Tschopp éd., Actes du Colloque international de Genève, septembre 2004
}

Pierre Fiala et Juliette Rennes

\section{(2) OpenEdition \\ Journals}

Édition électronique

URL : https://journals.openedition.org/mots/17073

DOI : $10.4000 /$ mots. 17073

ISSN : 1960-6001

Éditeur

ENS Éditions

Édition imprimée

Date de publication : 1 juillet 2006

Pagination : 55-60

ISBN : 2-84788-098-4

ISSN : 0243-6450

Référence électronique

Pierre Fiala et Juliette Rennes, « Marie-Claire Caloz-Tschopp éd., Actes du Colloque international de Genève, septembre 2004 », Mots. Les langages du politique [En ligne], 81 | 2006, mis en ligne le 01 juillet 2008, consulté le 24 avril 2022. URL : http://journals.openedition.org/mots/17073 ; DOI : https:// doi.org/10.4000/mots. 17073 
tissement. On pourrait aussi s'interroger sur le statut totémique de la langue nationale, au sens freudien de gardienne supérieure et identification de l'ordre social, tel que J. Widmer le conçoit dans ses modèles cognitifs, et ses relations avec cet étrange amour lacanien de la langue que Milner ${ }^{7}$ (1978) avait mis au jour naguère.

Autant de questions cruciales qui montrent l'intérêt de l'approche proposée et l'importance de ce travail où la Suisse apparait comme un laboratoire d'une balkanisation réellement en cours dans l'ensemble européen, et au niveau mondial. L'Harmattan, il est de bon ton de le dire dans les milieux académiques, publierait tout et n'importe quoi. C'est pourtant grâce à sa politique éditoriale que des idées neuves, souvent négligées chez les éditeurs de la notoriété, émergent et circulent sous une forme papier accessible. L'ouvrage de J. Widmer en est un nouvel exemple.

Pierre Fiala

Université Paris 12, Céditec

fiala@univ-paris12.fr

\section{Notes sur une participation à une expérience de recherche}

2004, Parole, pensée, violence dans l'État. Une démarche de recherche, $672 \mathrm{p}$. 2004, Contraintes, dilemmes, position des travailleurs du service public. Entretiens, $397 \mathrm{p}$.

2004, Le devoir de fidélité à l'État entre servitude, liberté, (in)égalité. Regards croisés, $455 \mathrm{p}$.

2005, L'action « tragique » des travailleurs du service public, $492 \mathrm{p}$.

Actes du Colloque international de Genève, septembre 2004

Marie-Claire Caloz-Tschopp éd. pour les quatre volumes

Paris, L'Harmattan

Une anthropologie politique de la notion de superfluité humaine doublée d'une lutte politique contre l'exclusion des travailleurs immigrés dans le libéralisme helvétique: l'expérience menée par le réseau animé par Marie-Claire CalozTschopp est originale et exemplaire. Commandée par le gouvernement genevois $^{8}$, avec un appui financier du Fonds national de la recherche scientifique

7. J.-C. Milner, 1978, L'amour de la langue, Paris, Le Seuil, $132 \mathrm{p}$.

8. Incarnée dans des figures historiques et des institutions, la République et Canton de Genève désignation officielle - cultive dans la longue durée son image de cité internationale et sa vocation universalisante : berceau de l'éthique protestante, du libéralisme politique et économique et de 
suisse, associant plusieurs facultés (Programme interdisciplinaire en action humanitaire, http://www.unige.ch/piah/) et l'Université ouvrière de Genève, cette recherche collective sur l'immigration est académique dans ses formes, politique dans ses finalités et son engagement. Un collectif de praticiens et de chercheurs s'est constitué il y a une dizaine années en un réseau de réflexion, d'interventions et de propositions qui s'efforce de répondre théoriquement et pratiquement aux questions actuelles soulevées par les politiques migratoires des pays développés d'Europe occidentale - de la Suisse en particulier ${ }^{9}$.

Les quatre épais volumes (2 000 pages, une soixantaine de contributeurs) rendent compte de cette recherche-action et en illustrent les caractères principaux. Ils comprennent des articles théoriques (juridiques, économiques, philosophiques, politologiques, sociologiques, linguistiques), des témoignages, des analyses de cas, des réflexions pratiques, mais aussi une série importante de «miroirs », documents de toutes natures (textes littéraires, philosophiques, sociaux, médiatiques ; iconographie artistique ou scientifique) qui, chemin faisant, de la pensée présocratique à celle de Hannah Arendt, Jürgen Habermas, Michel Foucault ou Cornelius Castoriadis, ont stimulé la réflexion collective. Après avoir présenté la démarche d'ensemble, nous revenons sur quelques questions, qui nous concernent plus directement ${ }^{10}$, soulevées par cette expérimentation politico-philosophique grandeur nature.

la puissance bancaire privée, siège de l'action humanitaire et sociale, de la recherche scientifique et du débat géopolitique. Calvin y imposa jadis les principes égalitaires et théocratiques de son Institution chrétienne, premier traité politico-religieux en français (1541). Rousseau, « citoyen d'un État libre », y conçut les principes du Contrat social fondés sur l'égalité politique. Voltaire, Necker, De Staël, Constant marquèrent durablement l'espace public de leurs influences personnelles. Dunant y établit concrètement les premiers droits des combattants et les bases de l'action humanitaire (1874). Denis de Rougemont y formula après la seconde guerre mondiale les principes d'un fédéralisme européen libéral, tandis que Jean Ziegler en faisait naguère la cible privilégiée de sa contestation de l'impérialisme bancaire. Des institutions scientifiques y ont développé les recherches expérimentales tant dans les sciences physiques que dans les sciences humaines et sociales. Le mini-espace public genevois revendique ainsi sa part d'universalisme à travers les variétés et les contrastes de l'innovation scientifique, politique et sociale.

9. Pour mesurer le chemin parcouru, il n'est pas inutile de comparer la présente entreprise à une recherche publiée en 1968 sous la direction de Jeanne Hersch, professeure de philosophie à l'université de Genève (Le droit d'être un homme, Paris, Unesco, Payot, 588 p.). Marqué par les pensées alors dominantes - existentialisme chrétien, structuralisme de Claude Lévi-Strauss -, ce recueil de textes et de documents iconographiques provenant des civilisations de toutes époques ouvrait une voie à la tolérance antiraciste en traitant concrètement de la genèse anthropologique des Droits de l'Homme dans des cultures parfois éloignées. Entre cette réflexion théorique et encyclopédique initiale sur l'émergence conceptuelle des droits dans l'histoire et le travail critique actuel du réseau, la rupture est nette. Il s'agit maintenant d'examiner pratiquement un problème politique crucial dans l'Europe des années 2000 : la remise en cause des droits humains fondamentaux (de déplacement, de travail, de résidence, d'association, de regroupement familial) dans une société qui tend à subordonner tous les droits aux règles du profit maximum.

10. Notre participation à cette expérience a consisté en une analyse des discours électoraux de la droite nationaliste helvétique dans ses rapports avec l'espace médiatique, qui est parue dans le troisième volume. De ce fait, notre présente contribution n'est pas à proprement parler un compte rendu, mais une réflexion issue d'une participation à un projet commun, sur quelques thèmes cruciaux de la politique migratoire sécuritaire européenne. Notre regard sur les quatre 
Le premier volume, Parole, pensée, violence dans l'État, entrelace l'exposé méthodologique des hypothèses philosophiques, institutionnelles et socioéconomiques qui ont conduit à recueillir la parole des agents de la fonction publique, administratifs, policiers, travailleurs sociaux, hospitaliers, chargés par l'État de " gérer » les flux migratoires et le chômage croissant, d'organiser en particulier les procédures de refoulement de la main-d'œuvre immigrée. Le second volume, Contraintes, dilemmes, position des travailleurs du service public, livre les entretiens sous deux formes, un corpus de vingt entretiens exhaustifs et des extraits thématiques regroupés dans un ordre raisonné selon trois positions des acteurs devant la place et la tâche qui leur sont assignées: adhésion, soumission ou résistance. Le troisième volume, Le devoir de fidélité à l'État entre servitude, liberté, (in)égalité. Regards croisés, présente quelques outils disponibles pour analyser de façon croisée les entretiens et leurs contextes. La problématisation se regroupe autour de cinq pôles: le devoir de fidélité dans le service public, les menaces actuelles sur la citoyenneté abordées à partir de la notion de total-libéralisme, la question des formes de la haine ou du mépris, celle des formes de la résistance, enfin la notion clé de la réflexion, la superfluité, issue de la pensée d'Hannah Arendt. Un quatrième volume, sous le titre L'action «tragique » des travailleurs du service public, recueille les travaux et les débats du colloque qui clôturait la recherche en septembre 2004. Ce dernier recueil est l'aboutissement d'une démarche qui vise notamment à transformer dans les faits, par l'exercice de la parole, les rapports aux étrangers (réfugiés, travailleurs, étudiants) des fonctionnaires chargés de "gérer» les politiques de surveillance, d'encadrement ou d'expulsion des étrangers. Ce personnel, dont le statut est lui-même précarisé par la réduction des moyens et des postes ou la privatisation des fonctions, se trouve en effet pris dans des situations ressenties de plus en plus comme «tragiques», contradictoires, voire violentes, que la parole permet parfois de transformer en résistance.

Dans son ensemble, l'ouvrage rappelle à certains égards le recueil édité en 1993 par Pierre Bourdieu ${ }^{11}$. Les deux ouvrages s'inscrivent dans une anthropologie de la parole sociale. Ils présentent l'un et l'autre une somme de témoignages, sous forme de données discursives transcrites scrupuleusement, dans leur logique et leurs formes langagières propres, sans céder pour autant à l'illusion réaliste. Ils réduisent la distance observateur-observé sans tomber pour autant dans la confusion des deux instances ni renoncer à commenter les témoignages langagiers, ni se placer du point de vue ethno-méthodologique. Ils refusent le

volumes est donc partiel. À défaut de rendre compte de l'ouvrage dans son ensemble, il met en cause le rapport entre recherche universitaire et intervention dans le domaine public, et entre réseau d'action, élaboration et expertise - autant de principes que partageait le collectif d'auteurs.

11. P. Bourdieu, 1993, La misère du monde, Paris, Le Seuil, 960 p. 
discours d'une expertise linguistique, sociologique ou philosophique en surplomb. Les différences sont néanmoins sensibles entre les deux entreprises. La publication bourdieusienne, essai abouti de sociologie du langage appliquée, est construite comme un itinéraire dramatisé, mettant à découvert, presque thêâtralement, la parole sociale en proie à la précarité et l'expression des contradictions politiques de nos sociétés. La recherche genevoise, éthique et politique, se présente davantage comme production intellectuelle en cours ${ }^{12}$, à facettes multiples, soulignant l'interaction permanente de la construction théorique et des pratiques à l'œuvre dans les institutions. La réflexion métapolitique fondée sur des analyses économiques approfondies, des données statistiques ${ }^{13}$, des schématisations graphiques, emprunte des cheminements esthétiques ${ }^{14}$ pour rejoindre la parole des acteurs eux-mêmes, tenter de dénouer dans l'échange les contradictions ressenties comme insupportables par les acteurs eux-mêmes et construire de nouvelles références de l'humain et de l'action politique. L'ouvrage s'appuie aussi sur des actes sociaux et politiques - campagnes contre des expulsions arbitraires, propositions d'amendements ou de législations nouvelles -, sur les structures d'intervention et les associations de résistance à la politique migratoire helvétique, désormais inscrite dans la doctrine européenne des cercles concentriques et illustrée par la politique sécuritaire de Schengen, appliquée par la Suisse avant même d'être entrée en vigueur dans l'Union européenne.

Plus encore que dans les enquêtes de P. Bourdieu, c'est la notion de dispositif, théorisée en particulier par M. Foucault, qui rend compte ici du travail :

Ce que j'essaie de repérer sous ce nom, c'est, premièrement, un ensemble résolument hétérogène, comportant des discours, des institutions, des aménagements architecturaux, des décisions réglementaires, des lois, des mesures administratives, des énoncés scientifiques, des propositions philosophiques, morales, philanthropiques, bref : du dit, aussi bien que du non-dit, voilà les éléments du dispositif. Le dispositif lui-même, c'est le réseau qu'on peut établir entre ses éléments. Deuxièmement, ce que je voudrais repérer dans le dispositif, c'est justement la nature du lien qui peut exister entre ces éléments hétérogènes [...]. Troisièmement, par dispositif, j'entends une sorte - disons - de formation, qui, à un moment historique donné a eu pour fonction majeure de répondre à une urgence. Le dispositif a donc une fonction stratégique dominante. (Foucault, 1994 ${ }^{15}$ )

12. Ces publications conservent dans leur écriture et leur facture les traces d'un travail en cours: absence d'index, hétérogénéité et finalisation parfois inégale des contributions, mais aussi oralité et simplicité des témoignages et des réflexions qui s’y rapportent.

13. D. Golaz, J. Mathey, G. A. Antille, «Les contraintes économiques du droit d'asile et du chômage sur les professionnels et les usagers », vol. 1, p. 421-549.

14. Une iconographie illustre la démarche, associant des images antiques du théâtre social, les formes de la catharsis et des figures héroïques, historiques et sociales de la résistance.

15. M. Foucault, 1994, Dits et écrits, 1954-1988, Paris, Gallimard, p. 299. Cité dans le vol. 1, p. 418. 
M. Foucault a décrit, on le sait, les aspects discursifs de ces dispositifs, sous le nom de formations discursives, mais sa méthode d'analyse, les étapes de la démarche et les données elles-mêmes, notamment les données discursives, sont de fait difficiles à reconstruire et à reproduire. L'expérience genevoise, elle, livre la progression collective de la construction des réseaux sémantiques et formels, à la fois hétérogènes, transdisciplinaires, historiquement actifs, objets permanents de controverses et d'enjeux. On y découvre le cheminement des agents sociaux dans l'interaction verbale des entretiens et l'évolution de leurs positions à travers l'explicitation de leurs expériences. Nous y reconnaissons pour notre part les éléments de ce que nous avons nommé les formations langagières (Ebel et Fiala, $1983^{16}$ ). L'analyse de la circulation des formules et de la transformation des lieux discursifs dans les pratiques langagières des agents et les discours institutionnels ou partidaires permettrait sans doute d'approfondir les représentations qui constituent l'imaginaire helvétique. Les trajets discursifs des agents, d'une part, et les diverses contributions, de l'autre, reconstituent déjà certaines stratégies du capitalisme bancaire helvétique qui gère les crises économiques, sociales ou militaires au plus près de ses intérêts. Souplement lié à l'Europe monétaire, mais solidement ancré et protégé au cœur de l'espace judiciaire et policier de Schengen, il accentue ses investissements mondiaux, réduit ses coûts intérieurs à l'aide d'une immigration sélectionnée et toujours ségréguée, diminue ses contributions au développement. Les contributions proposent ainsi des notions comme celles de libéralisme sécuritaire, de total-libéralisme ${ }^{17}$, de frontières intérieures, et placent en face d'elles les objectifs d'une égaliberté et des nouvelles civilités (É. Balibar, vol. 1) alternatives à celles d'équité, d'ingérence ou d'aide humanitaire, de commerce éthique ou d'aide au développement. Autre fil théorique : on suit le débat actuel sur la jetabilité (B. Ogilvie, vol. 1, p. 555), notion développée dans le cadre d'une critique anthropologique historique des droits humains, et qui désigne une gestion de la force de travail totalement disponible et la mise des corps sous surveillance totale. Notion qui s'appuie sur celle de superfluité humaine, avancée par H. Arendt dans le contexte génocidaire totalitaire, mais n'équivaut à elle ni par ses fondements, ni par sa portée, ni par son étendue, ni par son horreur (M.-C. Caloz-Tchopp, 2004). D'où la remarque de cette dernière, qui nous servira de conclusion :

Il est arrivé à plusieurs reprises que le Département fédéral de justice et police suisse invente des mythes et construise des dispositifs, des outils, pour les mettre en œuvre

16. M. Ebel et P. Fiala, 1983, Sous le consensus, la xénophobie. Paroles, arguments, contextes (19711981), Lausanne, Institut de science politique, $454 \mathrm{p}$.

17. "Une tension est à l'œuvre entre le régime/système politique de démocratie sécuritaire et un autre régime politique, que je nomme, à défaut d'un concept théorique existant, le régime de total-libéralisme. On peut se demander si le deuxième régime évoqué n'est pas en train d'advenir de manière sournoise derrière le premier. » (vol. 1, p. 373) 
(par exemple, outre le modèle des cercles, les empreintes digitales, le statut d'admission provisoire de réfugié de la violence, le système bonus-malus). Ces inventions, ces constructions réapparaissent ailleurs en Europe. La Suisse ne fait pas partie de l'Europe politique et, pourtant, on peut penser qu'elle est un laboratoire pour la construction des polices, la répression des populations en mouvement et trop timidement pour le crime organisé. (vol. 1, p. 372)

\section{Pierre Fiala}

Université Paris 12, Céditec

fiala@univ-paris12.fr

\section{Juliette Rennes}

Université Paris 12, Céditec jurennes@club-internet.fr 